From the Mayo Clinic, Rochester, MN.

Published online ahead of print at www.jco.org on November 18, 2013

Supported in part by the Mayo Clinic Hematological Malignancies Program and Paul Calabresi K12 Award (Grant No. CA90628), by Grants No. CA 107476, CA 62242, CA100707, and CA 83724 from the National Cancer Institute, and by the Jabbs Foundation and the Henry J. Predolin Foundation.

Authors' disclosures of potential conflicts of interest and author contributions are found at the end of this article.

Corresponding author: Shaji K. Kumar, MD, Professor of Medicine, Division of Hematology, Mayo Clinic, 200 First St SW, Rochester, MN 55905; e-mail:

kumar.shaji@mayo.edu.

(c) 2013 by American Society of Clinical Oncology

0732-183X/13/3136w-4529w/\$20.00

DOI: $10.1200 / J C O .2013 .49 .0086$

\title{
Importance of Achieving Stringent Complete Response After Autologous Stem-Cell Transplantation in Multiple Myeloma
}

Prashant Kapoor, Shaji K. Kumar, Angela Dispenzieri, Martha Q. Lacy, Francis Buadi, David Dingli, Stephen J. Russell, Suzanne R. Hayman, Thomas E. Witzig, John A. Lust, Nelson Leung, Yi Lin, Steven R. Zeldenrust, Arleigh McCurdy, Philip R. Greipp, Robert A. Kyle, S. Vincent Rajkumar, and Morie A. Gertz

Listen to the podcast by Dr Anderson at www.jco.org/podcasts

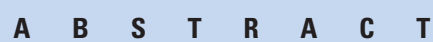

\section{Purpose}

To study the impact of achieving stringent complete response (sCR), an increasingly attainable goal, after autologous stem-cell transplantation (ASCT) in patients with multiple myeloma (MM).

\section{Patients and Methods}

Maximal response rates were determined in 445 consecutive patients who underwent ASCT within 12 months of diagnosis of MM. The patients achieving varying degrees of complete response (CR) are the focus of our study.

\section{Results}

One hundred and nine patients (25\%) achieved sCR after ASCT. The median overall survival (OS) rate from the time of transplantation for patients attaining $S C R$ was not reached (NR), in contrast to those patients achieving conventional complete response (CR; $n=37$; OS, 81 months) or near $\mathrm{CR}$ (nCR; $\mathrm{n}=91$; OS, 60 months; $P<.001$ ). Five-year OS rates were $80 \%, 53 \%$, and $47 \%$ for $\mathrm{sCR}$, $\mathrm{CR}$, and $\mathrm{nCR}$, respectively. The median time to progression (TTP) from ASCT of patients achieving sCR was significantly longer (50 months) than TTP of patients achieving CR or nCR (20 months and 19 months, respectively). On multivariable analysis, post-ASCT response of SCR was an independent prognostic factor for survival (hazard ratio, $0.44 ; 95 \% \mathrm{Cl}, 0.25$ to 0.80 ; versus $\mathrm{CR} ; P=.008$ ), in addition to proliferation rate, pre-ASCT cytogenetics, and performance status. OS rates of patients attaining SCR continued to remain superior at 2-year landmark (median, NR $v 70$ months for conventional CR group; $P=.007$ ).

\section{Conclusion}

Improved long-term outcome is seen after ASCT with achievement of sCR when compared with lesser degrees of responses. Myeloma trials reporting the response rates should identify patients achieving SCR and CR separately, owing to markedly disparate outcomes of the two categories.

\section{J Clin Oncol 31:4529-4535. (c) 2013 by American Society of Clinical Oncology}

\section{INTRODUCTION}

While multiple myeloma (MM) remains largely an incurable disease, the concept of operational cure, first proposed in patients who had sustained complete response (CR) beyond 10 years after high-dose therapy (HDT) with stem-cell support, is increasingly gaining ground. ${ }^{1-6}$ Deepening the magnitude of response to achieve operational cure in a proportion of MM patients, albeit small, is becoming an attainable goal with modern strategies using a combination of novel agents and autologous stem-cell transplantation (ASCT). ${ }^{7}$ However, owing to a paucity of clinical data, responses deeper than the tradi- tionally defined CR have not, until recently, been considered more consequential. ${ }^{8,9}$ Strategies focused on achieving deep responses have been questioned as achieving $\mathrm{CR}$ has not consistently translated into improved overall survival (OS). ${ }^{4,7,10-17}$ Moreover, substantial heterogeneity exists in the definitions of response-related end points for patients with MM. ${ }^{2,8,12}$ In clinical trials, the subset of patients achieving near-complete response (nCR), characterized by patients with less than $5 \%$ bone marrow plasma cells (BMPCs) and monoclonal protein detectable by immunofixation only, is often grouped with those who achieve standard CR (absence of monoclonal protein by electrophoresis and 
immunofixation along with less than 5\% BMPCs), ${ }^{18}$ based on an unsubstantiated assumption that the survival outcomes of patients in the two response categories are similar.

The development of International Myeloma Working Group (IMWG) uniform criteria for response is an endeavor to eliminate ambiguities in the response assessment and make cross-trial comparisons of efficacy simpler. ${ }^{6}$ The IMWG has created a newer, more rigorous response category of stringent complete response (sCR) because the current therapeutic strategies frequently lead to deeper responses. However, this category requires validation in clinical studies. We evaluated the impact of specific subcategories of CR (stringent CR, standard CR, and near CR/immunofixation-positive CR) and lesser degrees of responses on outcomes as measured by the time to progression (TTP) and OS of patients with MM undergoing early ASCT (ie, within 12 months of diagnosis) in the era of novel agents.

\section{PATIENTS AND METHODS}

Four hundred and forty-five prospectively followed patients with MM who underwent ASCT between September 2002 and December 2008 were assessed for their maximal response rates after ASCT. The start date was chosen to coincide with the routine availability of free light chain (FLC) assay for postASCT response assessment at our institution. The data were frozen for analyses in May 2012. The study was approved by the Mayo Clinic institutional review board and was performed in accordance with the Declaration of Helsinki.

The induction regimens used before ASCT varied. All patients had access to similar salvage therapies on relapse. Only responses after the first ASCT or planned tandem transplantations were considered. Maintenance therapy was generally not used after ASCT. Individual patient data of serum and urine protein electrophoresis, immunofixation, serum FLC assay, and bone marrow (BM) aspiration and biopsy obtained 60 days or later after ASCT were abstracted to determine the best response. Serum FLC assay (FREELITE, The Binding Site Ltd, Birmingham, United Kingdom) was performed by immunonephelometry using a commercial reagent set of polyclonal antibodies. The assay quantitated $\kappa$ and $\lambda$ free light chains, and a $\kappa / \lambda$ ratio was calculated. FLC ratios (rFLC) outside the 0.26 to 1.65 range were considered abnormal. ${ }^{19}$ Immunohistochemical studies with antibodies to $\kappa$ and $\lambda$ immunoglobulin light chains were performed on paraffin sections of the BM biopsy specimens. Clonality of plasma cells was additionally confirmed with the previously described slide-based plasma cell labeling index (PCLI) method. ${ }^{20}$

Response categories were determined in accordance with the IMWG uniform response criteria. A very good partial response (VGPR) was defined as $90 \%$ or greater reduction in serum M-component plus a 24 -hour urine M-component of less than $100 \mathrm{mg} .{ }^{6}$ Patients with less than 5\% BMPCs and unmeasurable M-proteins by electrophoresis but persistent serum and/or urine immunofixation were categorized separately as $\mathrm{nCR}$, though it should be emphasized that this response category has been incorporated into the larger VGPR category of the IMWG response criteria.

Patients with disappearance of any soft tissue plasmacytomas and BMPCs of less than $5 \%$ with negative immunofixation studies were considered to be in standard CR. Patients achieving CR for whom the involved FLC reduced sufficiently to normalize the $\operatorname{rFLC}(\kappa / \lambda$ range, 0.26 to 1.65$)$ in the absence of monoclonal BMPCs as assessed by immunohistochemistry or immunofluorescence were considered to have achieved sCR. The categories of partial remission (PR), stable disease (SD), and progressive disease (PD) were also used in accordance with the IMWG criteria. ${ }^{6}$ Survival curves were plotted by the Kaplan-Meier method and the differences were compared by logrank tests. ${ }^{21}$

The following prognostic factors were evaluated in a univariable analysis: age, performance status, serum creatinine, lactate dehydrogenase, $\beta_{2}-$ microglobulin, cytogenetics, pre- and post-transplantation response status, and PCLI. Factors significantly prognostic for OS in the univariate model $(P \leq$
.05) were studied in a multivariable analysis using a Cox proportional hazards model.

TTP was defined as the time from ASCT to disease progression; deaths as a result of causes other than disease progression were censored. ${ }^{22,23}$ OS was defined as the time from ASCT to death from any cause or last follow-up. We performed landmark analysis for response categories of sCR and CR at 2 years to ensure that all the patients achieving at least a CR had sufficient time to reach the response level being studied. Patients were categorized as having sustained $\mathrm{sCR}$ if the duration of sCR was at least 6 months. The statistical analysis was performed using JMP 9 software (SAS Institute, Cary, NC).

\section{RESULTS}

The baseline characteristics of the patients are listed in Table 1. Two hundred and eighteen patients (49\%) were alive at the time of analysis. The median time to ASCT was 6 months (range, 1.5 to 12 months) from diagnosis. The median estimated follow-up of patients was 77 months from ASCT (95\% CI, 73 to 82 months). Only a few patients achieving less than VGPR underwent a second transplantation ( $\mathrm{n}=$ $29)$ or received maintenance therapies $(n=12)$.

\begin{tabular}{|c|c|c|c|c|}
\hline Parameter & $\begin{array}{c}\text { No. of } \\
\text { Patients* }\end{array}$ & $\%$ & Median & Range \\
\hline $\begin{array}{l}\text { Age at transplantation, years } \\
\quad \geq 65\end{array}$ & 445 & 25 & 59 & $29-76$ \\
\hline Creatinine at transplantation, $\mathrm{mg} / \mathrm{dL}$ & 445 & & 1 & $0.4-10.1$ \\
\hline PCLI at transplantation, \% & 442 & & 0 & $0-11$ \\
\hline Patients with PCLI $\geq 3 \%$ & & 7 & & \\
\hline $\mathrm{LDH}, \mathrm{U} / \mathrm{L}$ & 431 & & 189 & $158-230 \dagger$ \\
\hline Patients with abnormal LDH (> 222 U/L) & & 29 & & \\
\hline CRP, mg/L & 425 & & 0.4 & $0.3-1.3+$ \\
\hline Serum $\beta_{2}$-microglobulin, $\mu \mathrm{g} / \mathrm{mL}$ & 442 & & 2.5 & $1.1-57$ \\
\hline $\begin{array}{l}\text { Serum creatinine, } \mathrm{mg} / \mathrm{dL} \\
\quad \geq 2\end{array}$ & & 7 & 1 & $0.4-10.1$ \\
\hline Durie-Salmon stage at diagnosis & 435 & & & \\
\hline 1 & & 0 & & \\
\hline $2 a$ & & 34 & & \\
\hline $2 b$ & & 3 & & \\
\hline $3 a$ & & 57 & & \\
\hline $3 b$ & & 6 & & \\
\hline Cytogenetics & 441 & & & \\
\hline Abnormal cytogenetics & & 15 & & \\
\hline Patients with bone disease & 445 & 85 & & \\
\hline ECOG performance status $<2$ & 441 & 91 & & \\
\hline Time from diagnosis to ASCT, months & 445 & & 6 & $1.5-12$ \\
\hline Pretransplantation response & 445 & & & \\
\hline sCR & 7 & 2 & & \\
\hline CR & 19 & 4 & & \\
\hline $\mathrm{nCR}$ & 29 & 7 & & \\
\hline VGPR $\ddagger$ & 51 & 11 & & \\
\hline PR & 207 & 47 & & \\
\hline SD & 96 & 22 & & \\
\hline PD & 36 & 8 & & \\
\hline \multicolumn{5}{|c|}{$\begin{array}{l}\text { Abbreviations: ASCT, autologous stem-cell transplantation; CR, complete response; } \\
\text { CRP, C-reactive protein; ECOG, Eastern Cooperative Oncology Group; LDH, lactate } \\
\text { dehydrogenase; nCR, near complete response; PCLI, plasma cell labeling index; PD, } \\
\text { progressive disease; PR, partial response; SCR, stringent complete response; SD, } \\
\text { stable disease; VGPR, very good partial remission. } \\
\text { *Total No. of patients for whom data were available at transplantation. } \\
\text { †Interquartile range. } \\
\text { ‡Excluding patients in nCR. }\end{array}$} \\
\hline
\end{tabular}




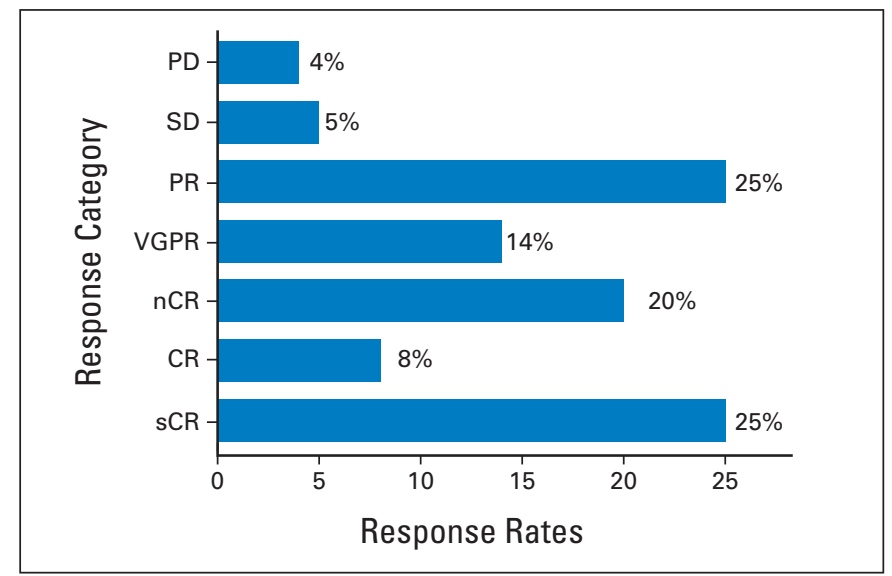

Fig 1. Best responses for 445 patients after autologous stem-cell transplantation. The response categories achieved by the patients were stringent complete response ( $\mathrm{sCR} ; \mathrm{n}=109$ ), complete response (CR; $n=37$ ), near complete response ( $n C R ; n=91$ ), very good partial response (VGPR [excluding $n C R$ ]; $n=$ 60), partial response (PR; $n=109$ ), stable disease (SD; $n=21$ ), and progressive disease (PD; $n=18$ ). The sum of percentages is slightly greater than 100 because of rounding.

\section{Response and Survival Outcomes}

One hundred and forty-six patients (33\%) achieved a response of CR or better after ASCT, including 109 patients (24\%) who achieved sCR. An additional 91 patients met the criteria for nCR. Eighteen patients (4\%) experienced disease progression despite ASCT (Fig 1). The TTP of patients who achieved CR or less after transplantation is shown by response categories in Figure 2A. No difference in TTP is noted among patients achieving responses ranging between SD and VGPR (including nCR). The median TTP is significantly longer (Fig 2B) for patients achieving sCR compared with $\mathrm{CR} / \mathrm{nCR}$.

The median OS of the entire cohort from the time of diagnosis was 83 months (95\% CI, 70 to 93). The median OS from diagnosis for patients achieving sCR was not reached (NR; 95\% CI, NR to
NR) versus 66 months for the rest of the cohort (95\% CI, 60 to 80; $P<.001)$.

The median OS from the time of transplantation of patients achieving at least a CR was 109 months (95\% CI, 94 to NR) compared with 64 months ( $95 \%$ CI, 53 to 78), 59 months (95\% CI, 50 to 86), and 56 months (95\% CI, 40 to NR), respectively, for patients achieving VGPR ( $\mathrm{n}=145 ; 33 \%)$, PR ( $\mathrm{n}=109 ; 24 \%)$, and SD ( $\mathrm{n}=21 ; 5 \%)$. Patients with PD survived for a median of 9 months after ASCT (Fig $3 \mathrm{~A})$. We then examined patients with varying categories of $\mathrm{CR}$, and found that patients with sCR have a significantly better outcome (median OS, NR; 5-year OS, 80\%). However, as with the TTP, no difference in OS is evident between patients achieving $\mathrm{CR}(\mathrm{n}=37$; $8 \%$ ) and nCR (immunofixation-positive CR; $n=91 ; 20 \%$; Fig 3B). The estimated 5-year OS is $80 \%$ (95\% CI, 72 to 87 ), 53\% (95\% CI, 38 to 67 ), and $47 \%$ ( $95 \% \mathrm{CI}, 37$ to 57 ) for patients achieving sCR, CR, and nCR, respectively.

Univariable and multivariable analyses for OS are listed in Table 2. Multivariable analysis was performed in 433 patients for whom pretransplantation data were available. Pretransplantation age, serum creatinine, and lactate dehydrogenase were not prognostic for OS on univariable analysis. Post-transplantation response status (sCR $v$ other inferior response categories), pretransplantation PCLI, cytogenetics, and Eastern Cooperative Oncology Group performance status were independent prognostic factors for OS in the multivariable analysis (Table 2).

Landmark analysis. Survival analysis was repeated using a 2-year landmark for the response categories of stringent CR and standard CR. OS of patients surviving at least 2 years from ASCT and achieving sCR continued to remain superior compared with the standard CR group (Fig 4A).

Among the patients achieving sCR, OS of patients with sustained sCR at 6 months from ASCT was not reached (5-year OS, 91\%; 7-year OS, $86 \%$ ) versus those who had nonsustained-sCR who had a median OS of 66 months (5-year OS, 53\%; 7-year OS, 37\%; $P<.001$; Fig 4B).
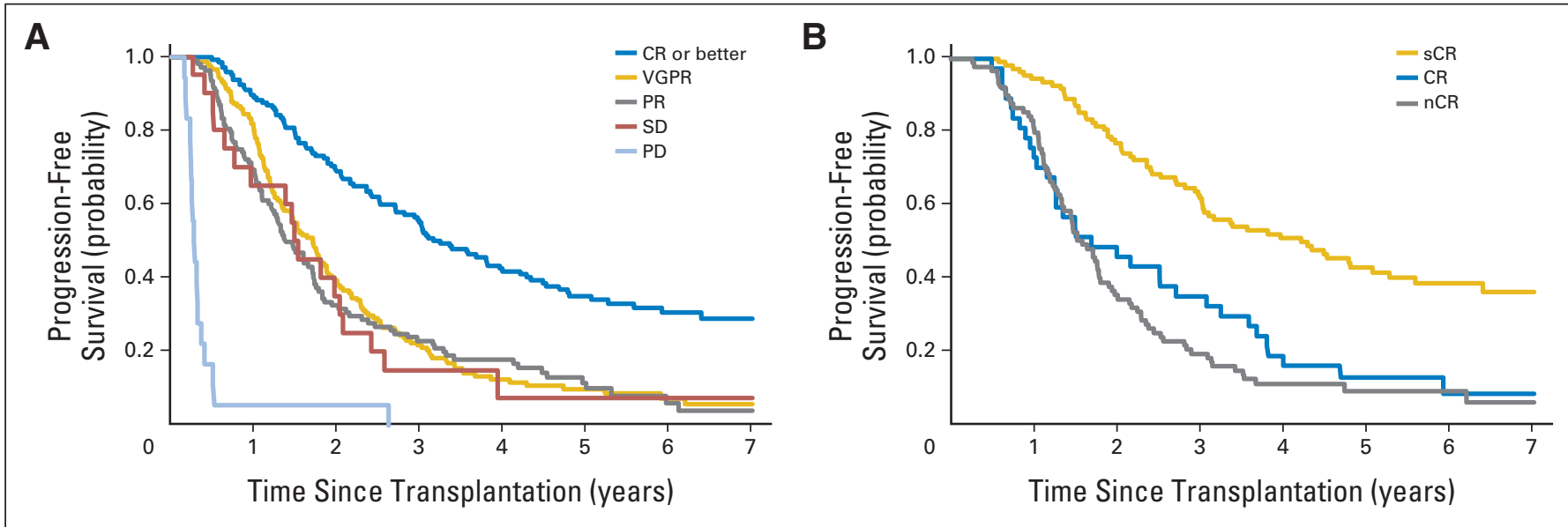

Fig 2. (A) The median time to progression (TTP) of patients achieving at least a complete response (CR; $n=146 ; 33 \%$ ) is 39 months ( $95 \%$ Cl, 33 to 48 ) compared

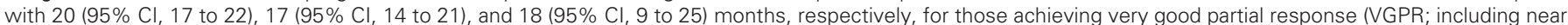

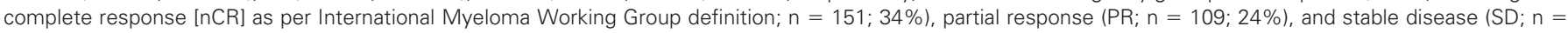

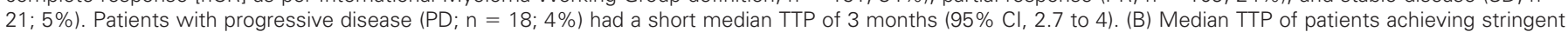

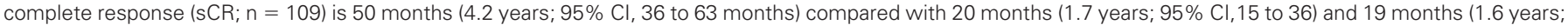
$95 \% \mathrm{Cl}, 16$ to 22 months) for groups attaining $\mathrm{CR}(\mathrm{n}=37)$ and $\mathrm{nCR}(\mathrm{n}=91)$, respectively $(P<.001)$. 

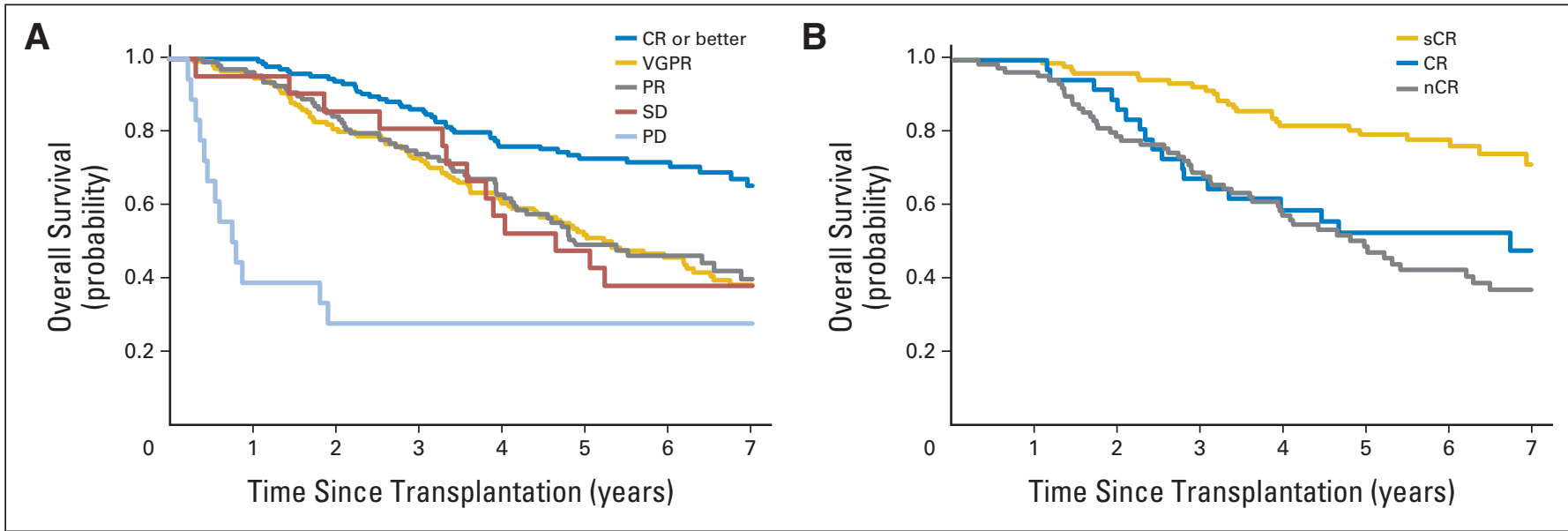

Fig 3. (A) Overall survival (OS) curves against response categories. The median OS of the patients achieving at least a complete response (CR; $n=146)$ was 109

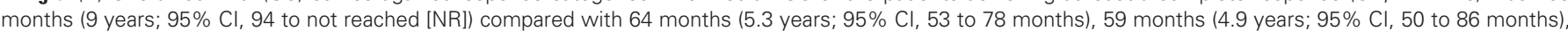

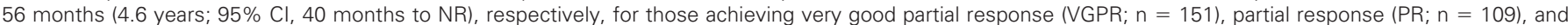

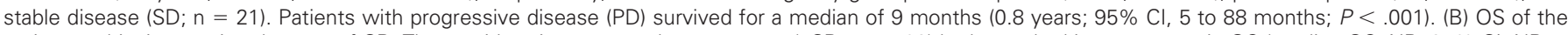

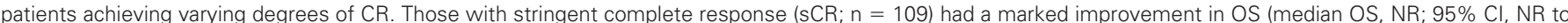

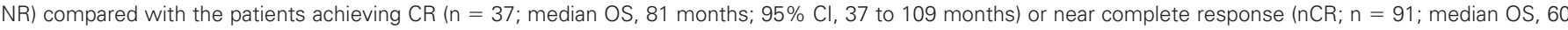
months; $95 \% \mathrm{Cl}, 47$ to 78$)$. The 5 -year OS is $80 \%, 53 \%$, and $47 \%$, for patients achieving sCR, CR, and nCR, respectively ( $P<.001)$.

\section{DISCUSSION}

With an increasing number of effective therapeutic options, the overarching debate about achieving cure versus control of MM has gained more relevance. ${ }^{2,4}$ The interest may be piqued, in part, because of a greater proportion of patients achieving deeper responses. ${ }^{24} \mathrm{CR}$ was until recently considered an uncommon $(<10 \%)$ and elusive outcome of traditional therapies. Following the initial observations of HDT by McElwain and Powles ${ }^{25}$, a larger study by Cunningham et $\mathrm{al}^{26}$ demonstrated achievement of CR in a third of patients, but neither

\begin{tabular}{|c|c|c|c|c|c|c|}
\hline \multirow[b]{2}{*}{ Parameter } & \multicolumn{3}{|c|}{ Univariate } & \multicolumn{3}{|c|}{ Multivariate $(n=433)$} \\
\hline & $\mathrm{HR}$ & $95 \% \mathrm{Cl}$ & $P$ & $\mathrm{HR}$ & $95 \% \mathrm{Cl}$ & $P$ \\
\hline \multicolumn{7}{|l|}{ Post-ASCT response } \\
\hline $\mathrm{sCR} \vee \mathrm{CR}$ & 0.37 & 0.21 to 0.67 & .001 & 0.44 & 0.25 to 0.80 & .008 \\
\hline $\mathrm{sCR} \vee \mathrm{nCR}$ & 0.30 & 0.18 to 0.46 & $<.001$ & 0.35 & 0.22 to 0.56 & $<.001$ \\
\hline sCR $\vee \vee G P R^{*}$ & 0.35 & 0.21 to 0.58 & $<.001$ & 0.42 & 0.25 to 0.70 & $<.001$ \\
\hline sCR $v$ PR & 0.34 & 0.21 to 0.53 & $<.001$ & 0.45 & 0.28 to 0.74 & .001 \\
\hline$s C R \vee S D$ & 0.30 & 0.16 to 0.60 & .001 & 0.33 & 0.16 to 0.67 & .003 \\
\hline$s C R \vee P D$ & 0.09 & 0.05 to 0.18 & $<.001$ & 0.14 & 0.06 to 0.28 & $<.001$ \\
\hline $\begin{array}{l}\text { Plasma cell labeling index, \% } \\
\quad \geq 3 v<3\end{array}$ & 5.02 & 3.29 to 7.36 & $<.001$ & 3.9 & 2.4 to 6.0 & $<.001$ \\
\hline $\begin{array}{l}\beta_{2} \text {-microglobulin, } \mu \mathrm{g} / \mathrm{mL} \\
\quad \geq 3.5 v<3.5\end{array}$ & 1.5 & 1.09 to 1.95 & .01 & 1.08 & 0.78 to 149 & .63 \\
\hline $\begin{array}{l}\text { Lactate dehydrogenase, } u / L \\
\text { Normal } v \text { abnormal }\end{array}$ & 0.75 & 0.57 to 1.01 & .06 & & & \\
\hline \multicolumn{7}{|l|}{ ECOG PS } \\
\hline$>1 \vee 0,1$ & 1.90 & 1.26 to 2.76 & .003 & 1.7 & 1.1 to 2.5 & .02 \\
\hline \multicolumn{7}{|l|}{ Cytogenetics } \\
\hline Normal $v$ abnormal & 0.48 & 0.35 to 0.67 & $<.001$ & 0.67 & 0.47 to 0.97 & .03 \\
\hline Pre-ASCT response & & & & 0.81 & 0.58 to 1.13 & .2 \\
\hline $\mathrm{PR} v<\mathrm{PR}$ & 0.57 & 0.44 to 0.74 & $<.001$ & & & \\
\hline $\begin{array}{l}\text { Age, years } \\
\quad \geq 65 v<65\end{array}$ & 0.99 & 0.73 to 1.33 & .95 & & & \\
\hline $\begin{array}{l}\text { Creatinine, } \mathrm{mg} / \mathrm{dL} \\
\quad \geq 2 \vee 2\end{array}$ & 1.03 & 0.58 to 1.67 & .92 & & & \\
\hline $\begin{array}{l}\text { Abbreviations: } A S C T \text {, autologo } \\
\text { hazard ratio; nCR, near complet } \\
\text { partial response. } \\
\text { *Excluding nCR. }\end{array}$ & $\begin{array}{l}\text { I trans } \\
\text { PD, pre }\end{array}$ & $\begin{array}{l}\text { R, complete } \\
\text { ease; PR, par }\end{array}$ & $\begin{array}{l}\text { COG PS, } \\
\text { e; sCR, st }\end{array}$ & $\begin{array}{l}\text { operat } \\
\text { olete r }\end{array}$ & $\begin{array}{l}\text { y Group perf } \\
\text {, stable disea }\end{array}$ & $\begin{array}{l}\text { us; } H R \text {, } \\
\text { ry good }\end{array}$ \\
\hline
\end{tabular}



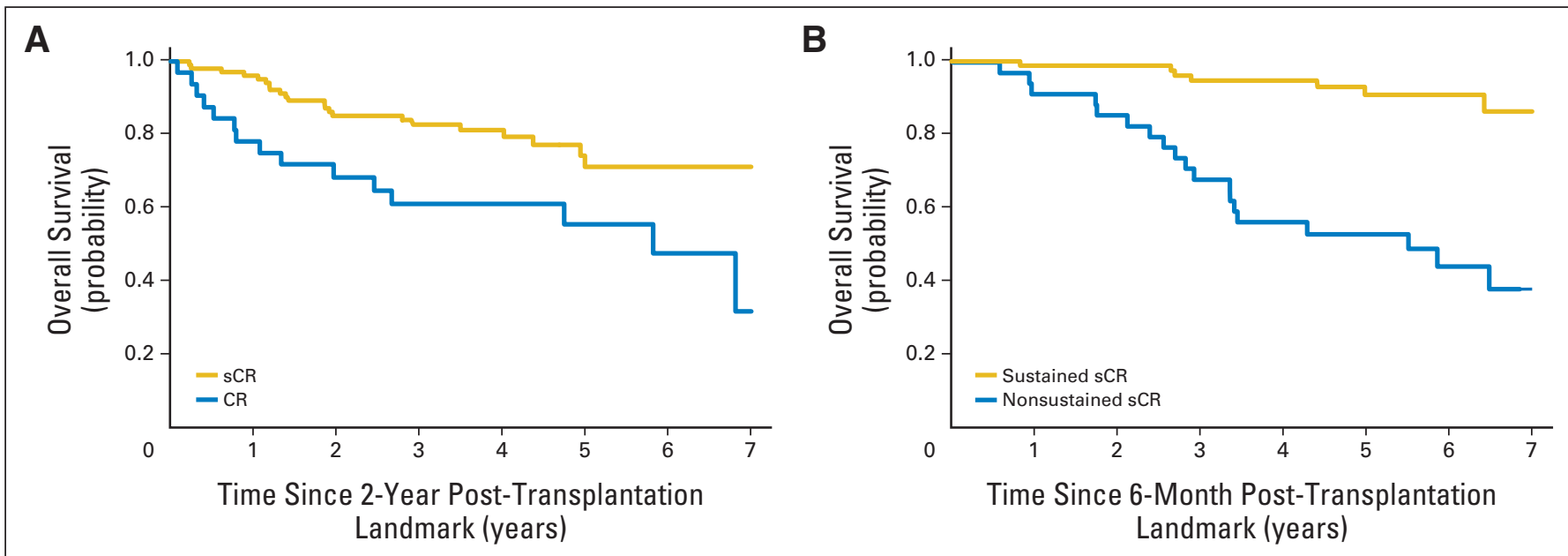

Fig 4. (A) Landmark analysis at 2 years studying survival outcomes of patients undergoing early autologous stem-cell transplantation (ASCT) and achieving stringent

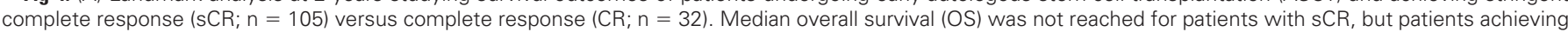

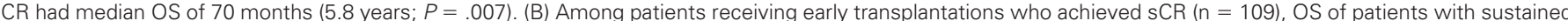

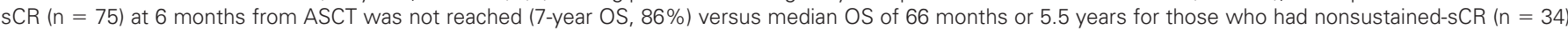
after ASCT (7-year OS, 37\%;P<.001).

survival-benefit nor durability of response was observed. Stem-cell rescue in subsequent studies permitted use of higher doses of melphalan, leading to CR in up to $40 \%$ to $60 \%$ of the patients. ${ }^{27,28}$ However, such remissions have not been sustained in most studies, and an eventual relapse of MM suggested persistent disease. It is therefore logical to assume that the use of more sensitive immunohistochemical or flow cytometric techniques and FLC assay to detect clonality, or the absence thereof, would provide superior assessments of response. It is conceivable that the application of such techniques would improve the precision of the response evaluation as supported by our study results.

A post hoc analysis of the Velcade As Initial Standard Therapy in Multiple Myeloma (VISTA) trial of bortezomib plus melphalanprednisone versus melphalan-prednisone demonstrated that achievement of $\mathrm{CR}$ in patients receiving the former therapy was associated with longer TTP (hazard ratio, $0.45 ; P=.004$ ) and a longer treatmentfree interval (hazard ratio, $0.39 ; P=.026$ ) compared with achievement of VGPR, but it was unknown whether teasing apart CR further had prognostic implications. ${ }^{29}$

Baseline serum FLC, a useful determinant of prognosis in MM, aids in the detection of low tumor burden that is generally undetected by conventional tests. Normalization of rFLC suggests restoration of polyclonality and is a prerequisite for $\mathrm{sCR}{ }^{6}$ Despite the lack of clarity in outcomes of those achieving responses deeper than CR, the IMWG recommends serial FLC assessment for documenting sCR during therapy. ${ }^{6,19}$ Although FLC response alone at 2 months with conventional alkylator-based therapies was predictive of subsequent M-protein response on serum protein electrophoresis, it failed to predict outcomes, and data with novel agents are scarce. ${ }^{30}$ The benefits of transplantation in further cytoreduction have been studied extensively in clinical trials, ${ }^{31,32}$ and therefore we restricted our analysis to the maximal post-transplantation responses. We focused on the maximal response obtained rather than a snapshot of response assessment on day 100 after ASCT. This strategy potentially helped eliminate false-positive results owing to discordant rFLC $(<0.26$ for $\kappa$ and $>1.65$ for $\lambda$ MM subtypes), which in most cases normalized over time.
Several recent studies have demonstrated that achieving CR translates into better outcomes, ${ }^{33,34}$ but our mature data set goes a step further, underscoring the importance of clonality assessments during follow-up. Indeed, such assessments by immunohistochemistry, immunofluorescence, and FLC have become part of routine clinical practice at most transplantation centers. Our study validates the inclusion of $\mathrm{sCR}$ in the IMWG criteria by demonstrating a significant improvement in TTP and OS with achievement of sCR compared with CR after ASCT, and underscoring the additional prognostic significance of restoration of polyclonality (seen with $\mathrm{sCR}$ ) beyond CR.

Multivariable analysis that incorporated other potential confounding factors, including pretransplantation response status, confirmed the independent high prognostic impact of achieving sCR post-transplantation for both OS (Table 2) and TTP (data not shown). However, our results require verification through prospective studies. Moreover, they cannot be extrapolated to the nontransplantation setting, though given the significant correlation of achieving CR with improved outcome, irrespective of age and ASCT eligibility in patients receiving novel agents, the results would probably be similar. Based on our findings, it is conceivable that obtaining responses of magnitude better than traditional CR in the early transplantation setting (provided a patient's quality of life is not compromised and the treatmentrelated toxicities are controlled) may alter the long-term disease course. However, it should be emphasized that only large randomized studies can ensure against biases, including the effect of unmeasured confounders.

It is equally important to emphasize that achieving sCR is but one marker of prognosis, a highly complex issue in MM. The interplay of several patient (host) and myeloma (disease biology) -related factors, including the durability of response, determine the outcome in individual patients. As such, our results highlighting the long-term benefits of maximal cytoreduction should be interpreted in context. A good proportion of our patients had received novel agents such as thalidomide $(\mathrm{n}=149)$, lenalidomide $(\mathrm{n}=103)$, and bortezomib $(\mathrm{n}=32)$ for induction. 
Notably, the definitions of sCR have somewhat differed in the studies conducted before the acceptance of the IMWG criteria. ${ }^{27,35}$ Although, our findings may seem to partly contradict a recent report in Journal of Clinical Oncology by Paiva et al, ${ }^{9}$ suggesting no difference between outcomes for patients with sCR and CR, the variation in the results are likely owing to the nonadherence in the latter study to the standard definition of the term sCR. In the Spanish study, sCR was defined as CR plus normal serum rFLC. The study did not mandate absence of clonality in the marrow as a prerequisite for achieving sCR.

The other important finding of equivalent survival outcomes in patients achieving lesser degrees of responses than CR merits further discussion. No survival difference is noted between patients achieving $\mathrm{nCR}$ and standard $\mathrm{CR}$ when those reaching sCR were categorized separately. Therefore, our study questions the prognostic value of further achieving immunofixation negativity after reaching $\mathrm{nCR}$ (ie, deepening response to standard CR) unless the rFLC normalizes and clonality disappears (ie, sCR is achieved). nCR and standard CR are two response categories merely separated by the detection of monoclonal protein on immunofixation, a test not infrequently riddled with subjective interpretation. ${ }^{36}$ Therefore, the comparable survival outcome of patients in these two categories in our study was not surprising. Indeed, sole reliance on this test has further come into question in a recent analysis of 295 patients on the GEM (Grupo Espanol de Mieloma) 2000 protocol. ${ }^{37}$ In this study, 31 patients who were immunofixation positive but minimal residual disease (MRD) negative by multiparameter flow cytometry had a significantly longer progression-free survival rate ( $65 v 37$ months) compared with those who were immunofixation-negative but MRD-positive on day 100 after ASCT. ${ }^{37}$ To what extent the long half-life of some immunoglobulins leading to the persistence of small clonal bands on immunofixation after ASCT could account for this discrepancy is unclear. Indeed, small amounts of abnormal protein bands (monoclonal proteins and/or oligoclonal bands) seen after an ASCT could represent recovery of the immune system and may be unrelated to the original malignant clone. Given that a substantial proportion of the patients in our study with CR went on to attain sCR (75\%), it is likely that the prognostic significance of CR seen in other studies so far is also primarily attributable to the proportion of patients with $\mathrm{sCR}$ rather than standard $\mathrm{CR}$.

Use of second transplantation or maintenance therapies, albeit in a few patients who achieved less than a VGPR, could possibly have favorably affected the outcomes, making them comparable to those attaining VGPR/nCR. Patients with SD performed just as well as those attaining PR or VGPR, confirming the findings of a recent large Spanish study. ${ }^{28} \mathrm{~A}$ possible explanation could be related to the inherent disease biology of such patients with a monoclonal gammopathy of undetermined significance-like state that allows them a long progression-free interval and OS. ${ }^{4} \mathrm{~A}$ study from Arkansas found that a fraction of 10-year myeloma survivors had never attained CR and the gene expression profiles of such patients bore characteristics similar to those seen in monoclonal gammopathy of undetermined significance. ${ }^{38}$ Patients with PD failed to achieve cytoreduction despite HDT and, understandably, fared the worst.

Our analysis focuses on determination of survival outcome based on the best response obtained after ASCT, and particularly highlights the significance of reaching varying degrees of CR. The results indicate that $\mathrm{sCR}$ represents a deeper response state compared with conventional CR, translating to longer duration of response after stem-cell transplantation (ie, longer TTP) and improved OS. However, a criti- cism of the current response criteria is the reliance on random BM biopsies to ascertain the depth of response. Response assessment with sensitive imaging techniques such as magnetic resonance imaging or positron emission tomography (not factored in our cohort as well) is not universally advocated, despite unfavorable implications of persistent focal lesions beyond clinical $\mathrm{CR}$, which can serve as potential sources for relapse. ${ }^{39}$

To overcome the inherent bias of studies comparing outcomes in responders and nonresponders (sCR $v$ no sCR), we performed a landmark analysis of patients who survived at least 2 years after ASCT. ${ }^{40}$ In this analysis, patients achieving sCR have a markedly superior outcome compared with those achieving standard CR. However, among patients achieving sCR, those with sustained-sCR achieve the best outcome. We chose a 6-month cutoff merely to highlight the adverse implications of poorly sustained $\mathrm{SCR}$.

An integrated approach with post-transplantation consolidation and maintenance therapies could permit attainment of more profound response states, including molecular remission. Although a few studies have attempted to illustrate the prognostic benefits of obtaining responses of such magnitude through the use of allele-specific oligonucleotide polymerase chain reaction, the practical application of this time-consuming and patient-specific primer-requiring test is limited. ${ }^{8}$ Multiparameter flow cytometry immunophenotyping to assess MRD also lacks widespread application currently. ${ }^{37}$ In contrast, our findings have substantial immediate clinical relevance owing to utilization of routinely available laboratory techniques.

In conclusion, our study demonstrates that post-ASCT sCR is a realistic, attainable goal and a surrogate for improved survival, and as such, it should be desirable and reported as a separate category in all myeloma-related clinical trials.

\section{AUTHORS' DISCLOSURES OF POTENTIAL CONFLICTS} OF INTEREST

Although all authors completed the disclosure declaration, the following author(s) and/or an author's immediate family member(s) indicated a financial or other interest that is relevant to the subject matter under consideration in this article. Certain relationships marked with a " $U$ " are those for which no compensation was received; those relationships marked with a " $C$ " were compensated. For a detailed description of the disclosure categories, or for more information about ASCO's conflict of interest policy, please refer to the Author Disclosure Declaration and the Disclosures of Potential Conflicts of Interest section in Information for Contributors. Employment or Leadership Position: None Consultant or Advisory Role: None Stock Ownership: None Honoraria: Morie A. Gertz, The Binding Site Group Research Funding: Angela Dispenzieri, Celgene, Millenium Pharmaceuticals, Janssen Pharmaceuticals, Pfizer Expert Testimony: None Patents: None Other Remuneration: None

\section{AUTHOR CONTRIBUTIONS}

Conception and design: Prashant Kapoor, Shaji K. Kumar, S. Vincent Rajkumar, Morie A. Gertz

Collection and assembly of data: Prashant Kapoor, Shaji K. Kumar, Angela Dispenzieri, Morie A. Gertz

Data analysis and interpretation: All authors

Manuscript writing: All authors

Final approval of manuscript: All authors 


\section{REFERENGES}

1. Barlogie B, Tricot GJ, van Rhee F, et al: Long-term outcome results of the first tandem autotransplant trial for multiple myeloma. $\mathrm{Br} J$ of Haematol 135:158-164, 2006

2. Hari $P$, Pasquini $M C$, Vesole DH: Cure of multiple myeloma: More hype, less reality. Bone Marrow Transplant 37:1-18, 2006

3. Mehta J, Singhal S: High-dose chemotherapy and autologous hematopoietic stem cell transplantation in myeloma patients under the age of 65 years. Bone Marrow Transplant 40:1101-1114, 2007

4. Rajkumar SV: Treatment of myeloma: Cure vs control. Mayo Clin Proc 83:1142-1145, 2008

5. Sirohi B, Powles R: International myeloma grand round. Lancet Oncol 2:571-579, 2001

6. Durie BG, Harousseau JL, Miguel JS, et al: International uniform response criteria for multiple myeloma. Leukemia 20:1467-1473, 2006

7. Usmani SZ, Crowley J, Hoering A, et al: Improvement in long-term outcomes with successive Total Therapy trials for multiple myeloma: Are patients now being cured? Leukemia 27:226-232, 2013

8. Ladetto M, Pagliano G, Ferrero S, et al: Major tumor shrinking and persistent molecular remissions after consolidation with bortezomib, thalidomide, and dexamethasone in patients with autografted myeloma. J Clin Oncol 28:2077-2084, 2010

9. Paiva B, Martinez-Lopez J, Vidriales MB, et al: Comparison of immunofixation, serum free light chain, and immunophenotyping for response evaluation and prognostication in multiple myeloma. $\mathrm{J}$ Clin Oncol 29:1627-1633, 2011

10. Alexanian $R$, Delasalle $K$, Wang $M$, et al: Curability of multiple myeloma. Bone Marrow Res 2012:916479, 2012

11. Cavo M, Tosi P, Zamagni E, et al: Prospective, randomized study of single compared with double autologous stem-cell transplantation for multiple myeloma: Bologna 96 clinical study. J Clin Oncol 25: 2434-2441, 2007

12. Chanan-Khan AA, Giralt S: Importance of achieving a complete response in multiple myelo$\mathrm{ma}$, and the impact of novel agents. J Clin Oncol 28:2612-2624, 2010

13. Facon T, Mary JY, Hulin C, et al: Melphalan and prednisone plus thalidomide versus melphalan and prednisone alone or reduced-intensity autologous stem cell transplantation in elderly patients with multiple myeloma (IFM 99-06): A randomised trial. Lancet 370:1209-1218, 2007

14. Fermand JP, Ravaud $P$, Chevret $S$, et al: High-dose therapy and autologous peripheral blood stem cell transplantation in multiple myeloma: Upfront or rescue treatment? Results of a multicenter sequential randomized clinical trial. Blood 92:31313136, 1998
15. Kumar SK, Dingli D, Lacy MQ, et al: Outcome after autologous stem cell transplantation for multiple myeloma in patients with preceding plasma cell disorders. Br J Haematol 141:205-211, 2008

16. Tricot G, Reiner M, Sawyer J, et al: A complete remission (CR) is not a prerequisite for prolonged survival after autotransplants for multiple myeloma. Blood 104, 2004 (abstr 926)

17. Dingli D, Pacheco JM, Nowakowski GS, et al: Relationship between depth of response and outcome in multiple myeloma. J Clin Oncol 25:49334937, 2007

18. Bladé J, Samson D, Reece D, et al: Criteria for evaluating disease response and progression in patients with multiple myeloma treated by high-dose therapy and haemopoietic stem cell transplantation: Myeloma Subcommittee of the EBMT-European Group for Blood and Marrow Transplant. $\mathrm{Br} J$ Haematol 102:1115-1123, 1998

19. Dispenzieri A, Kyle R, Merlini G, et al: International Myeloma Working Group guidelines for serum-free light chain analysis in multiple myeloma and related disorders. Leukemia 23:215-224, 2009

20. Greipp PR, Kumar S: Plasma cell labeling index. Methods Mol Med 113:25-35, 2005

21. Kaplan E: MP: Nonparametric estimation from incomplete observations. J Am Stat Assoc 53:457481, 1958

22. Rajkumar SV, Harousseau JL, Durie B, et al: Consensus recommendations for the uniform reporting of clinical trials: Report of the International Myeloma Workshop Consensus Panel 1. Blood 117: 4691-4695, 2011

23. Kumar SK, Lacy MQ, Dispenzieri A, et al: Early versus delayed autologous transplantation after immunomodulatory agents-based induction therapy in patients with newly diagnosed multiple myeloma. Cancer 118:1585-1592, 2012

24. Gay F, Larocca A, Wijermans P, et al: Complete response correlates with long-term progression-free and overall survival in elderly myeloma treated with novel agents: Analysis of 1175 patients. Blood 117: 3025-3031, 2011

25. McElwain TJ, Powles RL: High-dose intravenous melphalan for plasma-cell leukaemia and myeloma. Lancet 2:822-824, 1983

26. Cunningham D, Paz-Ares L, Milan $S$, et al: High-dose melphalan and autologous bone marrow transplantation as consolidation in previously untreated myeloma. J Clin Oncol 12:759-763, 1994

27. Barlogie B, van Rhee F, Shaughnessy JD Jr, et al: Making progress in treating multiple myeloma with total therapies: Issue of complete remission and more. Leukemia 22:1633-1636, 2008

28. Lahuerta JJ, Mateos MV, Martínez-LópezJ, et al: Influence of pre- and post-transplantation responses on outcome of patients with multiple myeloma: Sequential improvement of response and achievement of complete response are associated with longer survival. J Clin Oncol 26:5775-5782, 2008
29. Harousseau JL, Palumbo A, Richardson PG, et al: Superior outcomes associated with complete response in newly diagnosed multiple myeloma patients treated with nonintensive therapy: Analysis of the phase 3 VISTA study of bortezomib plus melphalanprednisone versus melphalan-prednisone. Blood 116: 3743-3750, 2010

30. Dispenzieri A, Zhang L, Katzmann JA, et al: Appraisal of immunoglobulin free light chain as a marker of response. Blood 111:4908-4915, 2008

31. Harousseau JL, Attal $\mathrm{M}$, Avet-Loiseau $\mathrm{H}$, et al: Bortezomib plus dexamethasone is superior to vincristine plus doxorubicin plus dexamethasone as induction treatment prior to autologous stem-cell transplantation in newly diagnosed multiple myeloma: Results of the IFM 2005-01 phase III trial. J Clin Oncol 28:4621-4629, 2010

32. Moreau $P$, Avet-Loiseau $H$, Facon $T$, et al: Bortezomib plus dexamethasone versus reduceddose bortezomib, thalidomide plus dexamethasone as induction treatment before autologous stem cell transplantation in newly diagnosed multiple myeloma. Blood 118:5752-5758, 2011

33. van de Velde HJ, Liu X, Chen G, et al: Complete response correlates with long-term survival and progression-free survival in high-dose therapy in multiple myeloma. Haematologica 92:1399-1406, 2007

34. Martinez-Lopez J, Blade J, Mateos MV, et al: Long-term prognostic significance of response in multiple myeloma after stem cell transplantation. Blood 118:529-534, 2011

35. Barlogie B, Hall R, Zander A, et al: High-dose melphalan with autologous bone marrow transplantation for multiple myeloma. Blood 67:1298-1301, 1986

36. Kyle RA, Rajkumar SV: Criteria for diagnosis, staging, risk stratification and response assessment of multiple myeloma. Leukemia 23:3-9, 2009

37. Paiva $B$, Vidriales MB, Cerveró J, et al: Multiparameter flow cytometric remission is the most relevant prognostic factor for multiple myeloma patients who undergo autologous stem cell transplantation. Blood 112:4017-4023, 2008

38. Zhan F, Barlogie B, Arzoumanian $V$, et al: Gene-expression signature of benign monoclonal gammopathy evident in multiple myeloma is linked to good prognosis. Blood 109:1692-1700, 2007

39. Barlogie B, Anaissie E, Haessler J, et al: Complete remission sustained 3 years from treatment initiation is a powerful surrogate for extended survival in multiple myeloma. Cancer 113:355-359, 2008

40. Hoering A, Crowley J, Shaughnessy JD Jr, et al: Complete remission in multiple myeloma examined as time-dependent variable in terms of both onset and duration in Total Therapy protocols. Blood 114:1299-1305, 2009 


\section{Acknowledgment}

We thank Shiv Lal Pandey, MBBS, and Utkarsh Painuly, MD, for their assistance with data collection. 